\title{
Adicción a las redes sociales en estudiantes mexicanos: percepciones de discentes y docentes
}

\author{
Rubicelia Valencia-Ortiz \\ Gerente de Innovación y Transformación Digital en Macmillan Education (Ciudad de México, México) \\ rubicelia.valencia@macmillaneducation.com | https://orcid.org/0000-0003-4656-5456
}

Julio Cabero-Almenara

Catedrático de Didáctica y Organización Escolar de la Universidad de Sevilla (España) cabero@us.es | https://orcid.org/0000-0002-1133-6031

Urtza Garay Ruiz

Profesora agregada de la Universidad del País Vasco (Bilbao, España) urtza.garay@ehu.eus | https://orcid.org/0000-0001-7298-9274

\section{Extracto}

La expansión de internet y los dispositivos móviles ha contribuido al cambio en las formas de comunicarnos. Actualmente, la mayoría de la población joven y adolescente está registrada y se comunica prioritariamente por medio de las redes sociales, lo que, en muchos casos, conlleva un uso abusivo y adictivo de las mismas. Esta investigación tiene como objetivo principal analizar las percepciones de estudiantes y docentes mexicanos respecto al nivel de adicción de los primeros a las redes sociales. La muestra la conforman 17.600 estudiantes y 1.480 docentes del nivel de preparatoria de México y el instrumento utilizado ha sido la social media addiction scale-student form (SMAS-SF), traducida, adaptada y validada al contexto mexicano. Entre los resultados, puede destacarse la existencia de diferencias significativas en las percepciones de ambos colectivos respecto a la adicción del alumnado a las redes sociales, así como a la frecuencia de uso y tiempo que invierten en ellas y en internet.

Palabras clave: redes sociales; adicción; población joven; alumnado; profesorado; adicción redes sociales; problemas redes sociales; satisfacción uso redes sociales.

Cómo citar: Valencia-Ortiz, R., Cabero-Almenara, J. y Garay Ruiz, U. (2021). Adicción a las redes sociales en estudiantes mexicanos: percepciones de discentes y docentes. Tecnología, Ciencia y Educación, 19, 103-122. https://doi.org/10.51302/tce.2021.616 


\title{
Addiction to social networks in Mexican students: perceptions of students and teachers
}

\author{
Rubicelia Valencia-Ortiz \\ Julio Cabero-Almenara \\ Urtza Garay Ruiz
}

\begin{abstract}
The expansion of the internet and mobile devices has contributed to a shift in communication. Currently, most youths and adolescents are registered and prioritize communication through social networks, which, in many cases, involves an abusive and addictive use of them. The main objective of this study is to analyze the perceptions that Mexican students and professors have regarding their level of addiction to social networks. The sample is made up of 17,600 students and 1,480 teachers in the Mexican high school system and the instrument used has been the Social Media Addiction Scale-Student Form (SMAS-SF) translated, adapted and validated to the Mexican context. Among the main results, it can be highlighted that there are differences between the perceptions of both groups regarding the addiction level of students to social networks as well as the frequency and time of social networking and navigating the internet.
\end{abstract}

Keywords: social networks; addiction; youth; students; teachers; social media addiction; social media problems; satisfaction with social media use.

Citation: Valencia-Ortiz, R., Cabero-Almenara, J. and Garay Ruiz, U. (2021). Addiction to social networks in Mexican students: perceptions of students and teachers. Tecnología, Ciencia y Educación, 19, 103-122. https://doi.org/10.51302/tce.2021.616 


\section{Sumario}

1. Introducción

2. Justificación

3. Diseño de investigación

\subsection{Objetivos}

3.2. La muestra

3.3. Instrumento de recogida de información

4. Resultados

5. Discusión de resultados

6. Conclusión

Referencias bibliográficas 


\section{Introducción}

La comunicación vía internet es una de las formas de interacción más usual actualmente sobre todo entre jóvenes y adolescentes. Rubio (2019), en un artículo con un sugerente título («La «generación muda»: los jóvenes que apenas llaman por teléfono»), indica que el $96,80 \%$ de las personas entre 14 y 24 años prefieren usar las aplicaciones de mensajería para comunicarse con familiares y amigos, más que el teléfono, y que utilizan a diario diferentes aplicaciones de las redes sociales. Este hecho ha llevado a algunos a hablar de que tal abuso puede ser un problema de salud pública (Malak et al., 2017).

\section{La comunicación vía internet es una de las formas de interacción más usual actualmente, sobre todo entre jóvenes y adolescentes}

Frente a las redes sociales presenciales, las electrónicas poseen diferentes peculiaridades: ausencia de contacto físico, inexistencia de relación entre la identidad del individuo en el mundo real (offline). El mundo online ofrece la posibilidad de relaciones sociales a aquellas personas que presentan dificultades para mantenerlas en directo. Las redes sociales son de fácil acceso, permiten la comunicación simultánea con un gran número de personas, facilitan el anonimato y hacen sencillo el proceso de romper o eliminar las relaciones o contactos (Fuentes et al., 2015).

En los últimos tiempos, su uso ha aumentado considerablemente entre la población joven y adolescente. Así lo han permitido las peculiaridades de las redes sociales señaladas anteriormente, referidas a la ausencia de contacto físico, inexistencia de relación entre la identidad del individuo en el mundo real y la fuerte presencia de los dispositivos móviles (García y Fernández, 2016; Gértrudix et al., 2017). En España, el 45,90 \% de los padres afirman que sus hijos han tenido el primer teléfono inteligente a los 14 años. De ellos, el 93,20\% lo utiliza como tecnología para conectarse a internet (Orange, 2018), mientras que el 85,10\% está registrado en al menos una red social (Rial et al., 2014).

Por lo general, el término «adicción» ha estado únicamente relacionado con el consumo de drogas y sustancias químicas, que es precisamente como se presenta en el Diagnostic and Statistical Manual of Mental Disorders formulado por la American Psychiatric Association (2013). La adicción se suele definir como un abuso y dependencia hacia cualquier tipo de sustancias psicoactivas (Basteiro et al., 2013). Sin embargo, distintos autores indican que el desarrollo de un proceso adictivo puede producirse sin la intervención de dichas sustancias, por lo que podría entonces definirse como un uso compulsivo y excesivo que 
provoca un deterioro en el funcionamiento diario del individuo y se convierte en una conducta adictiva (Watters et al., 2013), aunque no se puede hablar abiertamente ni de adicción a internet ni a las redes sociales, puesto que en el manual citado no se contempla así. Además, el uso excesivo de ciertas redes sociales como Facebook no es equivalente a un trastorno adictivo y algunos estudios plantean que se requieren más investigaciones al respecto (Castro y Moral, 2017).

Por tanto, no existe consenso en la literatura académica en cuanto al empleo del término «adicción» para referirse al consumo constante de internet y de las redes sociales. Pero, independientemente de la posición que se adopte, es innegable la existencia de un aumento progresivo en el uso de las tecnologías y en el acceso a ellas que tiene consecuencias negativas para las personas, en la medida en que genera trastornos en sus vidas (Fuentes et al., 2015) y, especialmente, en el colectivo vulnerable de la población adolescente (Ahmadi, 2014; Castro y Moral, 2017; Chóliz et al., 2012; Özdemir et al., 2014; Poli, 2017), un hecho que lo hace más preocupante, teniendo en cuenta que se encuentran en un periodo de formación cognitiva y emocional (Kuss y Griffiths, 2017).
No existe consenso en la literatura académica en cuanto al empleo del término «adicción» para referirse al consumo constante de internet y de las redes sociales. Pero, independientemente de la posición que se adopte, es innegable la existencia de un aumento progresivo en el uso de las tecnologías y en el acceso a ellas que tiene consecuencias negativas para las personas, en la medida en que genera trastornos en sus vidas

Para su diagnóstico, se han utilizado diferentes test de adicción a las redes sociales (TARS) (Ávila et al., 2018; Banyai et al., 2017; Basteiro et al., 2013; Escurra y Salas, 2014; Sahin, 2018; Simó et al., 2017; Tutgun-Ünal y Deniz, 2015), que suelen ser escalas de autopercepción con construcción tipo Likert. Últimamente, teniendo en cuenta el auge de estas investigaciones, han surgido una serie de metaanálisis que estudian y evalúan las diferentes escalas formuladas (Castro y Moral, 2017; Sigerson y Cheng, 2018).

\section{Justificación}

Asumiendo lo comentado, no se puede olvidar que un uso excesivo y abusivo de internet y de las redes sociales genera conductas y trastornos negativos, entre ellos: aislamiento social (Yu et al., 2016), distorsión entre el mundo real y digital (Llamas y Pagador, 2014), privación de sueño (Xanidis y Brignell, 2016), pérdida de interés para realizar otras acciones (Li et al., 2016), depresión (Boonvisudhi y Kuladee, 2017), problemas familiares (Fernández-Villa et al., 2015), baja autoestima (Lee y Cheung, 2014), problemas fisiológicos y de ansiedad creados por su abstinencia (Ahmadi, 2014; Kuss y Griffiths, 2017), fracaso escolar (Blachnio et al., 2016; Díaz-Vicario et al, 2019; Cañón et al., 2016; Malo-Cerrato et al., 
2018; Tartari et al., 2019; Tas, 2017), aumento de conductas de tipo violento, bullying y cyberbullying (Arnaiz et al., 2016; Martín et al., 2016; Martínez-Ferrer y Moreno, 2017) o problemas de salud (Li et al., 2016).

Por otra parte, desde la biología, se apunta que la fuerte e intensa presencia en internet produce cambios en la conectividad neuronal y en la estructura y funcionamiento del cerebro (He et al., 2017; Se-Hoon et al., 2016).

En el contexto mexicano, que es donde se centra nuestro estudio, han surgido una variedad de investigaciones que consolidan el uso elevado de internet y de las redes sociales como línea de investigación (Valerio y Serna, 2018). Además, y de acuerdo con la Asociación de Internet MX (2019), las redes sociales son la principal actividad realizada por los internautas mexicanos (82\%).

Finalizada la segunda década del siglo XXI, es indudable que el uso de las tecnologías se ha consolidado y forma parte de la cotidianidad de distintas generaciones. Tales tecnologías han tenido impactos destacables, pero estudios especializados han encontrado también aspectos preocupantes, especialmente por su uso excesivo y el potencial adictivo a ellas, que es el ámbito en el que se inserta el presente artículo y que se ha fortalecido en el contexto de un cambio de noción respecto a la adicción que los individuos pueden desarrollar a diversos comportamientos, una vez superada la idea de que dicho trastorno solo era posible por la inges-

Finalizada la segunda década del
siglo XXI, es indudable que el uso
de las tecnologías se ha consolidado
y forma parte de la cotidianidad
de distintas generaciones. Tales
tecnologías han tenido impactos
destacables, pero estudios
especializados han encontrado
también aspectos preocupantes,
especialmente por su uso excesivo
y el potencial adictivo a ellas
tión de sustancias psicoactivas (Andreassen et al., 2016). Este estudio es relevante por las conductas de riesgo que pueden derivarse del uso desmedido de las redes sociales e internet y que pueden afectar a los usuarios e incluso a terceras personas.

\section{Diseño de investigación}

\subsection{Objetivos}

Los objetivos perseguidos por este estudio de investigación son los siguientes:

- Analizar si existen diferencias significativas entre la percepción que tienen los estudiantes respecto a su adicción a las redes sociales y la percepción que tienen los docentes sobre el grado de adicción de su alumnado. 
- Analizar si existen diferencias significativas entre las percepciones tanto de estudiantes como de docentes con respecto a la frecuencia y al tiempo de uso de las redes sociales e internet por parte del alumnado.

Para el presente estudio, se desarrolló una investigación de tipo ex post facto (Mateo, 2004), ya que no se manipulan las variables estudiadas, sino que se accede a los datos tal como se encuentran en la realidad. En este tipo de estudios, el hecho ocurre primero y, posteriormente, se analizan las posibles causas y consecuencias, por lo que se trata de una investigación en la que no se modifica el fenómeno o situación objeto de análisis.

Para recoger la información (proceso en el que se profundiza más adelante), se empleó la escala social media addiction scale student form (SMAS-SF) de Sahin (2018), que fue traducida, adaptada y fiabilizada al contexto mexicano por Valencia y Castaño (2019).

\subsection{La muestra}

La población de esta investigación está compuesta por estudiantes y docentes que estudian y enseñan en el nivel de preparatoria de México. La muestra fue no probabilística, intencional o de conveniencia (Sabariego, 2012); se estableció según las necesidades requeridas por la investigación y se consideró a sujetos voluntarios que formaban parte de la población objeto de estudio. La muestra de estudiantes estuvo formada por 17.600 sujetos (5.451 hombres y 12.149 mujeres) y la de profesores por 1.498 individuos (596 hombres y 902 mujeres). Ambos colectivos pertenecían a las modalidades de estudio presencial (1.849 estudiantes y 276 docentes) y en línea (15.791 estudiantes y 1.222 tutores-facilitadores). La gran mayoría del alumnado tenía «menos de 20 años» $(f=4.101 ; 23,30 \%)$ y «entre 20 y 25 años» $(f=2.710 ; 15,40 \%)$.

\subsection{Instrumento de recogida de información}

La herramienta de diagnóstico, que como se ha señalado fue adaptada de Sahin (2018) al contexto mexicano, nos permite profundizar en el grado de adicción que el alumnado mexicano tiene respecto a las redes sociales y hace posible estudiar los objetivos planteados en esta investigación. Para esto se realizaron, vía internet, los cuestionarios para docentes y estudiantes en activo del nivel de preparatoria de México. El instrumento, elaborado con Google Forms, se administró vía internet durante el curso académico 2018-2019. Y los datos fueron analizados mediante el programa SPSS24.
La herramienta de diagnóstico nos permite profundizar en el grado de adicción que el alumnado mexicano tiene respecto a las redes sociales y hace posible estudiar los objetivos planteados en esta investigación 
La versión final de la escala quedó compuesta por 28 ítems con construcción tipo Likert y cinco opciones de respuesta (de 1 [totalmente en desacuerdo] a 5 [totalmente de acuerdo]). Los ítems se organizaron en torno a cuatro factores:

- Problemas. Consecuencias que se producen en el mundo social, laboral, académico, familiar y personal del sujeto como resultado del excesivo tiempo que la persona invierte en estar conectado a internet. Las actividades que el sujeto realiza en la red afectan negativamente en su vida.

- Satisfacción. Situación de placer que siente el individuo cuando se encuentra conectado a las redes sociales. El sujeto, cuando se encuentra conectado, vence sus problemas de obsesión y se siente aliviado.

- Obsesión de estar informado. Malestar que se produce en la persona por pensar que pueden estar ocurriendo cosas en internet y se las está perdiendo, y, por ello, siente una obsesión por estar siempre conectado, para no perderse nada de los que está sucediendo en el mundo. Por esta razón se produce la necesidad de estar conectado.

- Necesidad/obsesión de estar conectado. Malestar que se produce generalmente en el individuo cuando no se encuentra conectado y siente la necesidad de superar la abstinencia que sufre al no poder estar constantemente conectado a la red. Ello conduce al sujeto a pensar activamente en los medios sociales a los cuales está conectado y pasar de uno a otro constantemente.

El índice de fiabilidad del instrumento se obtuvo mediante el alfa de Cronbach y fue de 0,926, para el total de la escala; de 0,836, para el factor «Satisfacción»; de 0,827, para el factor «Problemas»; de 0,826, para el factor “Obsesión de estar informado»; y de 0,797, para el factor «Necesidad/obsesión de estar conectado». Valores todos ellos que indican índices altos de fiabilidad (Mateo, 2004).

\section{El índice de fiabilidad del instrumento se obtuvo mediante el alfa de Cronbach y fue de 0,926, para el total de la escala; de 0,836, para el factor «Satisfacción»; de 0,827, para el factor «Problemas»; de 0,826 , para el factor «Obsesión de estar informado»; y de 0,797, para el factor «Necesidad/obsesión de estar conectado»}

\section{Resultados}

Las puntuaciones medias y las desviaciones típicas del total de la escala, sus factores e ítems se presentan con todo detalle en el cuadro 1. 
Cuadro 1. Medias y desviaciones típicas en el total de la escala, en los factores y en los ítems

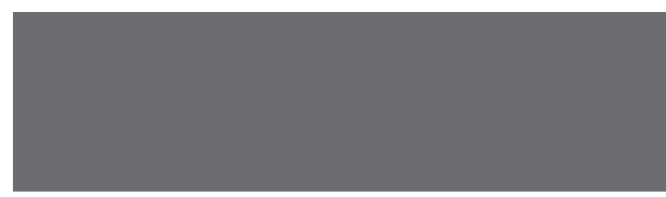

\begin{tabular}{|c|c|}
\hline \multicolumn{2}{|c|}{ Estudiantes } \\
\hline Media & $\begin{array}{c}\text { Desviación } \\
\text { típica }\end{array}$ \\
\hline
\end{tabular}

\begin{tabular}{|c|c|c|}
\multicolumn{2}{c}{ Docentes } \\
Media & $\begin{array}{c}\text { Desviación } \\
\text { típica }\end{array}$ \\
\hline
\end{tabular}

\begin{tabular}{lcccc} 
Total escala & 2,01 & 59 & 3,56 & 0,64 \\
\hline Dimensión 1. Problemas & 1,65 & 0,60 & 3,37 & 0,77 \\
\hline
\end{tabular}

Hasta mi familia se disgusta porque no puedo dejar de usar las redes sociales.

$\begin{array}{llll}1,87 & 1,04 & 3,60 & 0,95\end{array}$

Omito mis tareas porque paso mucho tiempo en las redes sociales.

$\begin{array}{llll}1,56 & 0,78 & 3,50 & 0,99\end{array}$

Me siento infeliz cuando no estoy en las redes sociales.

$\begin{array}{llll}1,81 & 1,04 & 3,46 & 0,96\end{array}$

Utilizo las redes sociales con tanta frecuencia que me olvido de mi familia.

$\begin{array}{llll}1,44 & 0,69 & 3,25 & 0,98\end{array}$

Ni siquiera noto que tengo hambre y sed cuando estoy en las redes sociales.

$\begin{array}{llll}1,42 & 0,71 \quad 3,07 & 1,07\end{array}$

Noto que mi productividad ha disminuido debido a las redes sociales.

$1,82 \quad 1,01 \quad 3,36 \quad 1,02$

Tengo problemas físicos debido al uso de las redes sociales.

$\begin{array}{llll}1,53 & 0,79 \quad 3,02 & 0,99\end{array}$

Uso las redes sociales incluso cuando voy por la calle para estar informado al instante sobre los acontecimientos.

$\begin{array}{llll}1,70 & 0,91 \quad 3,70 \quad 0,96\end{array}$

\begin{tabular}{lcccc}
\hline Dimensión 2. Satisfacción & $\mathbf{1 , 8 2}$ & $\mathbf{0 , 6 7}$ & $\mathbf{3 , 5 1}$ & $\mathbf{0 , 7 7}$ \\
\hline Una vida sin redes sociales no tiene sentido para mí. & 1,57 & 0,81 & 3,17 & 1,10 \\
\hline $\begin{array}{l}\text { Prefiero usar las redes sociales a pesar de estar } \\
\text { acompañado. }\end{array}$ & 1,73 & 0,85 & 3,66 & 0,98 \\
\hline $\begin{array}{l}\text { Prefiero las amistades de las redes sociales que } \\
\text { las presenciales. }\end{array}$ & 1,58 & 0,83 & 3,22 & 0,98 \\
\hline
\end{tabular}




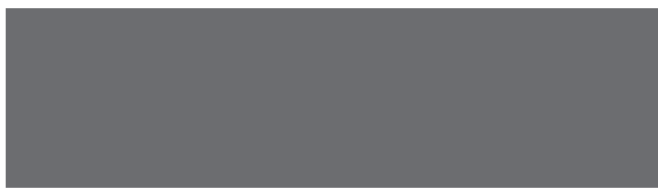

\begin{tabular}{|c|c|}
\multicolumn{2}{|c|}{ Estudiantes } \\
\hline Media & $\begin{array}{c}\text { Desviación } \\
\text { típica }\end{array}$ \\
\hline
\end{tabular}

\section{Docentes}

Media

Desviación típica

Me expreso mejor con las personas de las redes

2,01

1,03

3,54

1,00 sociales.

Aparento ser lo que yo quiero en las redes sociales.

1,65

0,89

3,70

0,95

Por lo general, prefiero comunicarme con personas a través de las redes sociales.

1,97

0,99

3,68

0,92

Prefiero comunicarme a través de las redes sociales para quedar en salir con alguien.

$2,24 \quad 1,15 \quad 3,59 \quad 0,97$

\begin{tabular}{|c|c|c|c|c|}
\hline Dimensión 3. Obsesión por estar informado & 2,34 & 0,75 & 0,63 & 0,61 \\
\hline Me emociona estar en las redes sociales. & 2,25 & 1,00 & 3,90 & 0,82 \\
\hline $\begin{array}{l}\text { El mundo misterioso de las redes sociales siem- } \\
\text { pre me cautiva. }\end{array}$ & 1,97 & 0,97 & 3,66 & 0,92 \\
\hline $\begin{array}{l}\text { Me gusta usar las redes sociales para mantenerme } \\
\text { informado sobre lo que sucede. }\end{array}$ & 3,00 & 1,16 & 3,79 & 0,89 \\
\hline $\begin{array}{l}\text { Navego en las redes sociales para mantenerme in- } \\
\text { formado sobre lo que comparten los grupos de me- } \\
\text { dios sociales. }\end{array}$ & 2,62 & 1,13 & 3,34 & 1,21 \\
\hline $\begin{array}{l}\text { Paso más tiempo en las redes sociales para ver } \\
\text { algunos eventos/acontecimientos especiales (por } \\
\text { ejemplo, cumpleaños, fiestas, etc.). }\end{array}$ & 2,07 & 1,01 & 3,66 & 0,91 \\
\hline $\begin{array}{l}\text { Mantenerme informado sobre las cosas relaciona- } \\
\text { das con mis cursos (tarea, actividades, etc.) me } \\
\text { hace estar siempre en las redes sociales. }\end{array}$ & 2,86 & 1,15 & 3,25 & 1,04 \\
\hline $\begin{array}{l}\text { Siempre estoy activo en las redes sociales para } \\
\text { estar inmediatamente informado sobre lo que com- } \\
\text { parten amigos y familia. }\end{array}$ & 2,27 & 1,05 & 3,86 & 0,83 \\
\hline
\end{tabular}




\begin{tabular}{|c|c|c|c|c|}
\hline & \multicolumn{2}{|c|}{ Estudiantes } & \multicolumn{2}{|c|}{ Docentes } \\
\hline & Media & $\begin{array}{l}\text { Desviación } \\
\text { típica }\end{array}$ & Media & $\begin{array}{l}\text { Desviación } \\
\text { típica }\end{array}$ \\
\hline \multicolumn{5}{|l|}{4} \\
\hline $\begin{array}{l}\text { Dimensión } 4 \text {. Necesidad/obsesión de estar co- } \\
\text { nectado }\end{array}$ & 2,01 & 0,86 & 3,92 & 0,80 \\
\hline Estoy ansioso por ir a las redes sociales. & 2,39 & 0,99 & 3,90 & 0,94 \\
\hline $\begin{array}{l}\text { Busco conectarme a internet en todas partes para } \\
\text { acceder a las redes sociales. }\end{array}$ & 2,28 & 1,06 & 4,03 & 0,97 \\
\hline $\begin{array}{l}\text { Los primero que hago cuando me despierto es co- } \\
\text { nectarme a las redes sociales. }\end{array}$ & 2,27 & 1,14 & 3,78 & 0,99 \\
\hline
\end{tabular}

Los resultados señalan diferentes aspectos:

- Las puntuaciones obtenidas por el profesorado superan el valor medio central de la escala de 2,5 y, en general, lo hacen 1,5 superior a las mostradas por el alumnado en el total de la escala y en los cuatro factores que la conforman.

- Las puntuaciones de las desviaciones típicas no son muy elevadas, tanto en estudiantes como en docentes, lo que sugiere cierta homogeneidad en las contestaciones.

- Para el profesorado, los estudiantes muestran cierta necesidad/obsesión por estar conectados e informados a través de las redes sociales. El alumnado, por su lado, no expresa esta preocupación.

Antes de analizar las hipótesis que se derivan de los objetivos formulados con anterioridad, deben señalarse cinco aspectos:

- Los análisis se efectúan, en primer lugar, para los datos obtenidos en la globalidad del instrumento y, posteriormente, para los alcanzados en cada uno de los cuatro factores identificados.

- Hemos utilizado los valores medios a efectos descriptivos porque son más fáciles de entender por el lector, al estar más familiarizado con los mismos.

- El estadístico que utilizaremos será la $t$-Student para muestras independientes, pero antes aplicaremos el estadístico de Levene para analizar la igualdad de las varianzas y, en función del resultado obtenido, determinar el valor $t$-Student que utilizaremos para su contraste. 
- Si se alcanzan diferencias significativas, obtendremos el tamaño del efecto mediante la $g$ de Hedges (Ellis, 2010) para conocer la potencia de tales diferencias.

- Los motivos de haber utilizado pruebas paramétricas frente a las no paramétricas son los siguientes: su potencia para rechazar o aceptar la hipótesis nula $(\mathrm{HO})$, ya que el amplio número de sujetos utilizados en la muestra reducía fuertemente la posibilidad de cometer los errores de tipo I y II a los que alude Siegel (1976), y la robustez de la prueba $t$-Student frente a desviaciones de la normalidad más que la aplicación del test de Levene para corregir el efecto de igualdad, o no, de las varianzas.

Las H0 que se contrastaron son: «No existen diferencias significativas entre la percepción del grado de adicción a las redes sociales mostrada (usos que hacen de internet, usos que hacen de las redes sociales, frecuencia de uso de las mismas y horas de utilización) por el alumnado y la mostrada por el profesorado respecto a sus estudiantes, con un riesgo alfa de equivocarnos del 0,05 ». Por el contrario, la hipótesis alternativa $(\mathrm{H} 1)$ hizo referencia a la existencia de tales diferencias.

En el cuadro 2, se presentan los valores obtenidos en el test de Levene y en la prueba estadística $t$-Student.

Cuadro 2. Valores $t$-Student para la existencia de diferencias significativas entre docentes y estudiantes respecto a la escala de adicción

\begin{tabular}{|l|c|c|c|c|c|}
\hline \multicolumn{7}{|c|}{ Test de Levene } \\
\hline & $F$ & Sig. & $t$ & $g l$ & Sig. \\
\hline Se asumen varianzas iguales & 3,610 & 0,057 & $-96,520$ & 19.096 & $0,000^{(* *)}$ \\
\hline Satisfacción & & & $-89,930$ & $1.719,449$ & 0,000 \\
\hline
\end{tabular}

Nota: $F$ = Estadístico Levene; Sig. = Significación; $t$ = Estadístico $t$-Student; $g l=$ Grados de libertad; ** = Significativo a 0,01 o inferior.

Fuente: elaboración propia.

Al no resultar significativo el test de Levene, se asume la igualdad de las varianzas y, en consecuencia, el valor $t$-Student que utilizaremos será $t=-96,520$, que para 1.719,449 grados de libertad es significativo a $p=0,000$, lo que permite rechazar la $\mathrm{HO}$ y aceptar la $\mathrm{H} 1$ con un nivel de significación $p \leq 0,001$.

Luego, podemos concluir que las percepciones que tienen estudiantes y docentes respecto al grado de adicción a las redes sociales son diferentes. $Y$ si observamos las medias presentadas en el cuadro 1, los docentes perciben que el alumnado tiene mayor nivel de adicción, en contraste con la percepción que tienen los estudiantes sobre sí mismos. 
Finalmente, analizamos el tamaño del efecto, cuyo valor es $g=2,504265$, que indica un efecto bastante elevado (Cohen, 1988).

En relación a la existencia o no de diferencias significativas en los cuatro factores, los valores obtenidos se presentan en el cuadro 3, donde se observa que, si se rechaza la $\mathrm{H} 0$, se presenta el valor $g$ del tamaño del efecto; a partir de este momento y, para no ser redundantes, solo presentaremos el valor $t$ seleccionado tras la aplicación del estadístico de Levene.

Cuadro 3. Valor $t$-Student y tamaño del efecto entre docentes y estudiantes para los cuatro factores de la escala de adicción

\begin{tabular}{|l|c|c|c|c|}
\multirow{2}{*}{ Factores } & \multicolumn{4}{c|}{$t$-Student/Tamaño del efecto } \\
\cline { 2 - 5 } & $t$ & $g l$ & Sig. & $g$ \\
\hline Problemas & $-84,537$ & $1.656,338$ & 0,000 & 2,4962 \\
\hline Satisfacción & $-82,622$ & $1.700,035$ & 0,000 & 2,3423 \\
\hline Obsesión por estar informado & $-71,371$ & $1.901,792$ & 0,000 & 1,7506 \\
\hline Necesidad/obsesión de estar conectado & $-72,754$ & $1.806,764$ & 0,000 & 1,8947 \\
\hline
\end{tabular}

Nota: $t$ = Estadístico $t$-Student; $g l=$ Grados de libertad; Sig.= Significación; $g$ = Tamaño del efecto.

Fuente: elaboración propia.

Los valores obtenidos permiten rechazar en todos los casos la $\mathrm{HO}$ y aceptar la $\mathrm{H} 1 \mathrm{a} \mathrm{p}=$ $=0,000$. Por tanto, se concluye que se dan diferencias significativas entre las opiniones que los estudiantes y los docentes tienen respecto a su grado de adicción en cuanto a «Problemas», «Satisfacción por estar conectados», «Obsesión por estar informado» y «Necesidad/ obsesión de estar conectado». El alumnado muestra percepciones más bajas en relación a esos cuatro factores analizados.

Como ocurrió con la puntuación global de la escala, aquí, los valores $g$ obtenidos para cada factor $(2,4962,2,3423,1,7506$ y 1,8947) sugieren efectos bastante elevados (Cohen, 1988).

\section{A) Usos que hacen de internet}

En el cuadro 4, presentamos lo que indicaron los estudiantes sobre los usos de internet y las percepciones que los docentes tenían de dicha utilización. 
Cuadro 4. Medias y desviaciones típicas de estudiantes y docentes respecto a los usos de internet por el alumnado

\begin{tabular}{|c|c|c|c|}
\hline & & Media & $\begin{array}{l}\text { Desviación } \\
\text { típica }\end{array}$ \\
\hline \multirow{2}{*}{ Redes sociales (Facebook, Instagram, etc.). } & Estudiantes & 2,65 & 1,080 \\
\hline & Docentes & 3,40 & 0,709 \\
\hline \multirow{2}{*}{ Mensajería instantánea (Facebook, Skype, etc.). } & Estudiantes & 2,45 & 1,138 \\
\hline & Docentes & 3,09 & 0,833 \\
\hline \multirow{2}{*}{ Chats o grupos (WhatsApp, Telegram, etc.). } & Estudiantes & 2,87 & 1,119 \\
\hline & Docentes & 3,55 & 0,657 \\
\hline \multirow{2}{*}{ Navegar libremente por diferentes páginas web. } & Estudiantes & 2,57 & 0,993 \\
\hline & Docentes & 2,89 & 0,795 \\
\hline \multirow{2}{*}{ Buscar información que me piden para mis estudios. } & Estudiantes & 3,27 & 0,797 \\
\hline & Docentes & 2,69 & 0,935 \\
\hline \multirow{2}{*}{$\begin{array}{l}\text { Buscar y descargar diferentes cosas (música, imá- } \\
\text { genes, documentos, etc.). }\end{array}$} & Estudiantes & 2,57 & 1,053 \\
\hline & Docentes & 3,02 & 0,780 \\
\hline \multirow{2}{*}{ Ver películas o series directamente, sin descargar. } & Estudiantes & 1,84 & 1,286 \\
\hline & Docentes & 2,61 & 0,873 \\
\hline \multirow{2}{*}{ Escuchar música o ver vídeos sin descargar. } & Estudiantes & 2,52 & 1,165 \\
\hline & Docentes & 2,99 & 0,816 \\
\hline \multirow{2}{*}{ Juegos en línea. } & Estudiantes & 0,81 & 1,124 \\
\hline & Docentes & 2,40 & 1,050 \\
\hline
\end{tabular}

Fuente: elaboración propia.

De nuevo, para analizar si había diferencias significativas, se aplicó la t-Student. También, en el caso de rechazar la $\mathrm{H} 0$, se obtuvo el tamaño del efecto, con el objeto de comprobar el valor de las diferencias encontradas. En todos los casos se rechazaron las $\mathrm{HO}$ formuladas a un nivel de $p \leq 0,001$ : «Redes sociales (Facebook, Instagram, etc.)» ( $t=-37,550$; $g=0,71017)$; «Mensajería instantánea (Facebook, Skype, etc.)» $(t=-27,701 ; g=0,57291)$; 
"Chats o grupos (WhatsApp, Telegram, etc.)» $(t=-35,664 ; g=0,62392)$; «Navegar libremente por diferentes páginas web» ( $t=-14.597 ; g=0,32688)$; «Buscar información que me piden para mis estudios» ( $t=23,134 ; g=0,71722)$; «Buscar y descargar diferentes cosas (música, imágenes, documentos, etc.)» $(t=-20,888 ; g=0,43511)$; «Ver películas o series directamente sin descargar» $(t=-31,422 ; g=0,611182)$; «Escuchar música o ver vídeos sin descargar» $(t=-31,422 ; g=0,100729)$; $y$ «Juegos en línea» $(t=-52,898 ; g=1,42170)$.

En todos los casos, rechazamos las $\mathrm{HO}$ a un nivel de significación de $p \leq 0,01$; luego, se concluye, que la percepción del uso que hacen de internet los estudiantes varía entre ambos colectivos (los mismos estudiantes y sus docentes). Solo en el caso de «Buscar información que me piden para mis estudios», los docentes que trabajaban en la preparatoria en línea consideraron que sus estudiantes hacían un mayor uso de internet. En el resto de ítems, los docentes que trabajaban de manera presencial consideraron que los estudiantes utilizaban la red en mayor grado que los alumnos que lo hacían de manera a distancia.

Las puntuaciones permiten señalar grandes efectos del tamaño en el ítem «Juegos en línea»; efectos intermedios o moderados en «Redes sociales (Facebook, Instagram, etc.)», «Mensajería instantánea (Facebook, Skype, etc.)», «Chats o grupos (WhatsApp, Telegram, etc.)» y «Buscar información que me piden para mis estudios»; y efectos bajos en «Buscar y descargar diferentes cosas (música, imágenes, documentos, etc.)», «Ver películas o series directamente sin descargar», «Escuchar música o ver vídeos sin descargar» y «Navegar libremente por diferentes páginas web».

\section{B) Usos que hacen de las redes sociales}

En cuanto a si había diferencias significativas entre las percepciones que los estudiantes y los docentes tenían de los usos de las redes sociales por el alumnado, también, en este caso, se rechazaron todas las HO a $p \leq 0,001$ : «Recibir información» $(t=-15,53 ; g=0,385)$, "Comunicarse con los amigos y familia» $(t=-31,24 ; g=0,584)$, «Estudio y formación» $(t=13,91 ; g=0,350)$ y «Conocer personas» $(t=-55,952 ; g=1,176)$.

Con los valores alcanzados, rechazamos todas las $\mathrm{H} 0$ formuladas, que hacían referencia a la no existencia de diferencias significativas con un riesgo alfa de equivocarnos del 0,01 . Se concluyó que los estudiantes y los docentes tienen percepciones diferentes respecto a los niveles de utilización de las redes sociales por el alumnado.

En general, el profesorado piensa que los estudiantes utilizan las redes sociales en un nivel alto para «Recibir información», "Comunicarse con amigos y familia» y «Conocer personas». En contrapartida, los estudiantes piensan que utilizan más las redes sociales para «Estudio y formación». Sobre el tamaño de los efectos, los valores se sitúan en pequeños («Recibir información» y «Estudios y formación»), intermedios («Recibir información») y elevados («Conocer personas»), en función del valor estadístico encontrado. 


\section{C) Frecuencia y horas de utilización de las redes sociales}

Respecto a la frecuencia y a las horas de utilización, los resultados obtenidos permitieron rechazar las $\mathrm{HO}$ a $p \leq 0,001$ : «Frecuencia con que participa en las redes sociales» $(t=-32,33-g=$ $=0,662)$ y «Horas de utilización semanalmente de las redes sociales» $(t=-32,83-g=0,915)$. Luego, podemos concluir que existen diferencias significativas entre las percepciones que los estudiantes y los docentes tienen respecto a la frecuencia con que los primeros participan en las redes sociales y el número de horas que las utilizan a la semana. Los docentes perciben que los estudiantes las utilizan con más frecuencia y durante más horas. El tamaño del efecto fue «intermedio» en la frecuencia de participación y «grande» en las horas.

\section{Discusión de resultados}

Los resultados obtenidos indican, en primer lugar, una fuerte diferencia entre la percepción que los estudiantes tienen respecto a su adicción a las redes sociales y la que sus docentes expresan. Por lo general, los estudiantes creen que los usos que hacen de las redes sociales no sugieren ni adicción ni otro tipo de riesgo, mientras que el profesorado opina lo contrario. Según los estudiantes, las actividades y el tiempo que interaccionan en las redes sociales no les crea «problemas» ni en el mundo sociolaboral ni en el académico; no les conduce a una situación de extrema «satisfacción» por el placer que les despierta el estar conectados; no sienten la «obsesión" por saber qué está ocurriendo en las redes sociales; y no presentan un malestar que les lleva a una «necesidad/obsesión» por estar siempre conectados a ellas. Por el contrario, los docentes opinan que sus estudiantes utilizan varias veces las redes sociales (74,37\%), una cifra que duplica la percepción que muestran los estudiantes (46,82\%). Dato similar se obtiene respecto al número de horas, ya que, uniendo las opciones de respuesta, «entre 20-25 horas» y «más de 25 horas», los estudiantes se posicionan en el $19 \%$ y los docentes en el $32 \%$. En síntesis, podemos decir que las diferencias entre ambos colectivos son significativas y bastante elevadas.

Aunque las investigaciones que han relacionado las percepciones de estudiantes y docentes sobre la adicción a las redes sociales son más bien limitadas, las que se han llevado a cabo coinciden con los resultados aquí obtenidos (Fasli y Ozdamli, 2018). Por ejemplo, Sánchez-Rodríguez et al. (2015), así como Marín-Díaz et al. (2019) han indicado que los estudiantes no perciben que tengan una conducta nociva en las redes sociales. Sin embargo, la elevada frecuencia y el número de horas que pasan en las redes sociales, que es una variable explicativa de la adicción a las mismas (Castro y Moral, 2017; Hunt et al., 2018; Kuss y Griffiths, 2017), sugieren lo contrario.

Estas diferencias se muestran también en los usos que el alumnado hace de internet, donde, en todos los casos, se encontraron diferencias entre las percepciones mostradas por el alumnado y el profesorado. Por lo general, los docentes creen que sus estudiantes hacen más uso de internet, con fuertes diferencias en «Juegos en línea», «Redes sociales» y «Chatear». La única diferencia se observó en «Buscar información que me piden para mis estudios», donde los estudiantes opinan que lo usan mucho, mientras los docentes piensan lo contrario. 


\section{Conclusión}

A partir de este análisis, puede afirmarse que existen diferencias significativas entre la percepción que los docentes y los estudiantes tienen sobre los usos de internet y de las redes sociales por parte de estos últimos. El profesorado percibe que sus estudiantes utilizan estas herramientas en mayor grado, a diferencia de la percepción que el alumnado tiene. Tales diferencias se han mantenido tanto en el caso de los docentes en línea como en los presenciales. Las diferencias de percepciones encontradas también se muestran en la frecuencia y en el tiempo de uso tanto de las redes sociales como de internet. En todos los usos se encontraron diferencias entre las percepciones expresadas por los estudiantes y por los docentes.

Tal situación, más el hecho de que los estudiantes aprenden a utilizar las redes sociales mediante «ensayo y error» (Tejada et al., 2019), sugiere la necesidad de establecer acciones formativas en los centros educativos con la finalidad de que los estudiantes adquieran una competencia digital para hacer un uso adecuado de las redes sociales, de forma que se prevengan las conductas de riesgo apuntadas. En este sentido, varios autores, entre ellos Fong et al. (2016), han elaborado modelos formativos para que los estudiantes dominen distintos aspectos de las tecnologías, como son la búsqueda, la evaluación y la organización de la información, así como la colaboración, el análisis, la presentación y la protección.

Como en toda investigación, esta que hemos presentado tiene algunas limitaciones, entre ellas, el hecho de que se utilizó un instrumento de diagnóstico de conductas y acciones basado en percepciones de los propios sujetos, es decir, no recoge información directa, sino a través de la valoración que hacen las personas, quienes pueden no ser conscientes de la realización de sus acciones o de la influencia de determinados fenómenos y acontecimientos. Como líneas futuras de investigación, se sugiere replicar este estudio en otros niveles de enseñanza, como en la educación secundaria o universitaria, y observar el grado en el que los hallazgos se mantienen. Esto significaría su consolidación desde una perspectiva conceptual.

\section{Referencias bibliográficas}

Ahmadi, K. (2014). Internet addiction among Iranian adolescents: a nationwide study. Acta Médica Iránica, 52(6), 467-472.

American Psychiatric Association. (2013). Diagnostic and statistical manual of mental disorders: DSM-IV-TR.

Andreassen, C. S., Billieux, J., Griffiths, M. D., Kuss, D. J., Demetrovics, Z., Mazzoni, E. y Pallesen, S. (Marzo 2016). The relationship between addictive use of social media and video games and symptoms of psychiatric disorders: a large-scale cross-sectional study. Psychology of Addictive Behaviors, 30(2), 252-262. https://doi.org/10.1037/ad b0000160

Arnaiz, P., Cerezo, F., Giménez, A. y Maquilón, J. (2016). Conductas de ciberadicción y experiencias de cyberbullying entre adolescentes. Anales de Psicología, 32(3), 761-769. https://doi.org/10.6018/analesps.32.3. 217461 
Asociación de Internet MX. (2019). 20 años. https://www.asociaciondeinternet.mx/es/

Ávila Berrio, D., Pardo Jaime, E. L. y Muñoz Rojas, M. ${ }^{a}$ L. (2018). Escala de adicción al internet de Lima (EAIL): análisis psicométrico. Revista Iberoamericana de Psicología, 11(3), 100-107. https://doi.org/10.33881/2 027-1786.rip.11309

Banyai, F., Asila, A., Király, O., Maraz, A., Elekes, Z., Griffiths, M. y Demetrovics, Z. (2017). Problematic social media use: results from a large-scale nationally representative adolescent sample. PLOS ONE, 12(1), 1-13.

Basteiro, J., Robles, A., Juarros, J. y Pedrosa, I. (2013). Adicción a las redes sociales: creación y validación de un instrumento de medida. Revista de Investigación y Divulgación en Psicología y Logopedia, 3(1), 2-8.

Blachnio, A., Przepiorka, A. y Pantic, I. (2016). Association between Facebook addiction, self-estem and life satisfaction: a cross-sectional study. Computers in Human Behavior, 55, 701-705. https://doi.org/10.1016/j. chb.2015.10.026

Boonvisudhi, T. y Kuladee, S. (2017). Association between internet addiction and depression in Thai medical students at Faculty of Medicine. RamathibodiHospital. PLOSONE, 12(3), 1-10. https://doi.org/10.1371/journal. pone.0174209

Cañón Buitrago, S. D., Castaño Castrillón, J. J., Hoyos Monroy, D. C., Jaramillo Hernández, J. C., Leal Ríos, D. R., Rincón Viveros, R., Sánchez Preciado, E. A. y Ureña Calderón, L. E. (2016). Uso de internet y su relación con la salud en estudiantes universitarios de la ciudad de Manizales (Caldas-Colombia). 20152016. Archivos de Medicina, 16(2), 312-325.

Castro Gerónimo, A. y Moral Jiménez, M. ${ }^{a}$ V. (2017). Uso problemático de redes sociales 2.0 en nativos digitales: análisis bibliográfico. Health and Addictions/Salud y Drogas, 17(1), 73-85.
Chóliz, M. y Marco Puche, C. (2012). Adicción a internet y redes sociales: tratamiento psicológico. Alianza Editorial.

Cohen, J. (1988). Statistical Power Analysis for the Behavioral Sciences. Erlbaum.

Díaz-Vicario, A., Mercader Juan, C. y Gairín Sallán, J. (2019). Uso problemático de las TIC en adolescentes. Revista Electrónica de Investigación Educativa, 21, 1-11.

Ellis, P. D. (2010). The Essential Guide to Effect Sizes: Statistical Power, Meta-Analysis, and the Interpretation of Research Results. Cambridge University Press.

Escurra Mayaute, M. y Salas Blas, E. (2014). Construcción y validación del cuestionario de adicción a redes sociales (ARS). LIBERABIT, 20(1), 73-91.

Fasli, E. y Ozdamli, F. (2018). Teacher candidates' opinions regarding instructional and safe use of social networks and internet addiction risk levels. TEM Journal, 7(2), 405410. https://doi.org/10.18421/TEM72-23

Fernández-Villa, T., Aguacil Ojeda, J., Almaraz Gómez, A., Cancela Carral, J. M. . , DelgadoRodríguez, M., García-Martín, M., JiménezMejías, E., Llorca, J., Molina, A. J., Ortiz Moncada, R., Valero-Juan, L. F. y Martín, V. (2015). Uso problemático de internet en estudiantes universitarios: factores asociados y diferencias de género. Adicciones, 27(4), 265-275. https://doi.org/10.20882/ adicciones.751

Fong, B., Lo, M. F. y Ng, A. (2016). A theoretical model to integrate PKM with Kolb's learning model for mitigating risks from exhaustive internet exposures. Electronic Journal of Knowledge Management, 14(3), 166-176.

Fuentes, J. L., Esteban, F. y Caro, C. (2015). Vivir en internet: retos y reflexiones para la educación. Síntesis.

García Galera, M. ${ }^{a}$ C. y Fernández Muñoz, C. (Coords.). (2016). Si lo vives, lo compartes. Cómo se comunican los jóvenes en un mundo digital. Fundación Telefónica-Ariel. 
Gértrudix Barrio, M., Borges, E. y García García, F. (2017). Vidas registradas. Redes sociales y jóvenes en la era algorítmica, Telos, 107, 62-70.

He, Q., Turel, O. y Bechara, A. (2017). Brain anatomy alterations associated with social networking site (SNS) addiction. Scientific Reports, 7. https://doi.org/10.1038/serp45 064

Hunt, M., Marx, R., Lipson, C. y Young, J. (2018). No more FOMO: limiting social media decreases loneliness and depression. Journal of Social and Clinical Psychology, 37(10), 751-768. https://doi.org/10.1521/ jscp.2018.37.10.751

Kuss, D. J. y Griffiths, D. (2017). Social networking sites and addiction: ten lessons learned. International Journal of Environmental Research and Public Health, 14, 1-17. https://doi.org/10.3390/ijerph14030311

Lee, Z. y Cheung, C. (2014). Problematic use of social networking sites: the role of selfesteem. International Journal of Business and Information, 9(2), 143-159. https://pdfs. semanticscholar.org/37cb/ca2ee1526bfe 276b52cd1c500186651f632c.pdf

Li, W., O'Brien, J. E., Snyder, S. M. y Howard, M. O. (2016). Diagnostic criteria for problematic internet use among U. S. university students: a mixed-methods evaluation. PLOS ONE, 11(1). https://doi.org/10.1371/ journal.pone.0145981

Llamas Salguero, F. y Pagador Otero, I. (2014). Estudio sobre las redes sociales y su implicación en la adolescencia. Enseñanza \& Teaching, 32(1), 43-57.

Malak, M. Z., Khalifeh, A. H. y Shuhaiber, A. H. (2017). Prevalence of internet addiction and associated risk factors in Jordanian school students. Computers in Human Behavior, 70, 556-563. https://doi.org/10.1016/j.chb. 2017.01.011

Malo-Cerrato, S., Martín-Perpiñá, M. ${ }^{a}$ y ViñasPoch, F. (2018). Uso excesivo de redes sociales: perfil psicosocial de adolescentes españoles. Comunicar. Revista Cientifica de Comunicación y Educación, 26(56), 101110. https://doi.org/10.3916/C56-2018-10

Marín-Díaz, V., Vega-Gea, E. y Passey, D. (2019). Determination of problematic use of social networks by university students. RIED. Revista Iberoamericana de Educación a Distancia, 22(2), 135-152. https://doi. org/10.5944/ried.22.2.23289

Martín Montilla, A., Pazos Gómez, M. ${ }^{a}$, Montilla Coronado, M. ${ }^{a}$ V. C. y Romero Oliva, C. (2016). Una modalidad actual de violencia de género en parejas de jóvenes: las redes sociales. Educación XXI, 19(2), 405-429. http://dx.doi.org/10.5944/educxx1.16473

Mateo Andrés, J. (2004). La investigación ex post-facto. En R. Bisquerra Alzina (Ed.), Metodología de la investigación educativa (pp. 195-230). La Muralla.

Martínez-Ferrer, B. y Moreno Ruiz, D. (2017). Dependencia de las redes sociales virtuales y violencia escolar en adolescentes. International Journal of Developmental and Educational Psychology. INFAD. Revista de Psicología, 1(1), 105-114. https://doi.org/ 10.17060/ijodaep.2017.n1.v2.923

Orange. (2016). Guía sobre el uso responsable de la tecnología en el entorno familiar.

Özdemir, Y., Kuzucu, Y. y Ak, Ş. (2014). Depression, loneliness and internet addiction: how important is low self-control? Computers in Human Behavior, 34, 284-290. https://doi. org/10.1016/j.chb.2014.02.009

Poli, R. (2017). Internet addiction update: diagnostic criteria, assessment and prevalence. Neuropsychiatry, 7(1), 4-8.

Rial, A., Gómez, P., Braña, T. y Varela, J. (2014). Actitudes, percepciones y uso de internet y las redes sociales entre los adolescentes de la comunidad gallega (España). Anales de Psicología. 30(2), 642-655. https://doi.org/ 10.6018/analesps.30.2.159111

Rubio, I. (2 de abril de 2019). La «generación muda»: los jóvenes que apenas llaman por 
teléfono. El País. https://elpais.com/tecnolo gia/2019/04/02/actualidad/1554220116_ 828140.html

Sabariego Puig, M. (2012). El proceso de investigación. En R. Bisquerra Alzina (Ed.), Metodología de la investigación educativa (pp. 127-163). La Muralla.

Şahin, C. (2018). Social media addiction scalestudent form: the reliability and validity study. TOJET. The Turkish Online Journal of Educational Technology, 17(1), 168-182.

Sánchez-Rodríguez. J., Ruiz-Palmero, J. y Sánchez-Rivas, E. (2015). Uso problemático de las redes sociales en estudiantes universitarios. Revista Complutense de Educación, 26 (número especial: T/C en Educación), 603-618.

Se-Hoon, J., HyoungJee, K., Jung-Yong, Y. y Yoori, H. (2016). What type of content are smartphone users addicted to?: SNS vs. games. Computers in Human Behavior, 54, 10-17. https://doi.org/10.1016/j.chb.2015. 07.035

Siegel, S. (1976). Estadística no paramétrica. Trillas.

Sigerson, L. y Cheng, C. (2018). Scales for measuring user engagement with social network sites: a systematic review of psychometric properties. Computers in Human Behavior, 83, 87-105. https://doi.org/10.1016/j.chb.2 018.01 .023

Simó Sanz, C., Martínez Sabater, A., Ballester Tarín, M. L. y Domínguez Romero, A. (2017). Instrumentos de evaluación del uso problemático del teléfono móvil/smatphone. Salud y Drogas, 17(1), 5-14.

Tartari, E., Tartari, A. y Bashiri, D. (2019). The involvement of students in social network sites after their learning. International Journal of Emerging Technologies in Learning (iJET), 14(13), 33-46. https://doi.org/ 10.3991/ijet.v14i13.10453

Taş, I. (2017). Relationship between internet addiction, gaming addiction and school engagement among adolescents. Universal Journal of Educational Research, 5(12),
2.304-2.311. https://doi.org/10.13189/ujer. 2017.051221

Tejada Garitano, E., Castaño Garrido, C. y Romero Andonegui, A. (2019). Los hábitos de uso en las redes sociales de los preadolescentes. RIED. Revista Iberoamericana de Educación a Distancia, 22(2),119-133. https://doi.org/10.5944/ried.22.2.23245

Turel, O., He, Q., Xue, G., Xiao, L. y Bechara, A. (Diciembre 2014). Examination of neural systems sub-serving Facebook «addiction». Psychological Reports, 115(3), 675-695. https://doi.org/10.2466/18.PR0.115c31z8

Tutgun-Ünal, A. y Deniz, L. (2015). Development of the social media addiction scale. AJIT-e: Online Academic Journal of Information Technology, 6(2)1, 51-70.

Valencia Ortiz, R. y Castaño Rodríguez, C. (2019). Use and abuse of social media by adolescents: a study in Mexico. Píxel-Bit. Revista de Medios y Educación, 54, 7-28. https://doi.org/10.12795/pixelbit.2019.154.01

Valerio Ureña, G. y Serna Valdivia, R. (2018). Redes sociales y bienestar psicológico del estudiante universitario. Revista Electrónica de Investigación Educativa, 20(3), 19-28. https://doi.org/10.24320/redie.2018.20. 3.1796

Watters, C. A., Keefer, K. V., Kloosterman, P. H., Summerfeldt, L. J. y Parker, J. D. A. (2013). Examining the structure of the internet addiction test in adolescents: a bifactor approach. Computers in Human Behavior, 29(6), 2.294-2.302. https://doi.org/10.1016/ j.chb.2013.05.020

Xanidis, N. y Brignell, C. M. (2016). The association between the use of social network sites, sleep quality and cognitive function during the day. Computers in Human Behavior, 55, 121-126. https://doi.org/10.1016/j. chb.2015.09.004

Yu, S., Wu, A. y Pesigan, I. (2016). Cognitive and psychosocial health risk factors of social networking addiction. International Journal of Mental Health and Addiction, 14(4), 550-564. https://doi.org/10.1007/s1 1469-015-9612-8 